\title{
Methylation regulation of liver-specific microRNA-122 expression and its effects on the proliferation and apoptosis of hepatocellular carcinoma cells
}

\author{
T.J. Xing, H.T. Xu, W.Q. Yu and D.F. Jiang \\ Department of Infectious Diseases, Taizhou People's Hospital, Taizhou, China \\ Corresponding author: T.J. Xing \\ E-mail: xingtj518@sina.com
}

Genet. Mol. Res. 12 (3): 3588-3597 (2013)

Received October 2, 2012

Accepted February 28, 2013

Published September 13, 2013

DOI http://dx.doi.org/10.4238/2013.September.13.3

\begin{abstract}
The regulation mechanism and significance of microRNA-122 (miRNA-122) expression are unclear. The aim of this study was to investigate the effects of DNA methylation on liverspecific miRNA-122 expression, cell proliferation, and apoptosis in hepatocellular carcinoma. Methylation of the miRNA-122 promoter region was detected through methylation sequencing. The level of miRNA-122 expression was measured using real-time quantitative polymerase chain reaction. The proliferation and apoptosis of hepatocellular cell lines were detected using flow cytometry and Cell Counting Kit-8 assays. Compared with those in human primary hepatocytes, methylation levels of the miRNA-122 promoter in the Huh7, HepG2, and QSG-7701 cell lines were significantly increased $(\mathrm{P}=0.000)$. Similarly, levels of miRNA-122 expression in these cell lines significantly decreased $(\mathrm{P}=0.007)$. After treatment with 5-aza-2deoxycytidine, the Huh7 and HepG2 cell lines displayed a significantly lower degree of methylation $(\mathrm{P}=0.038$ and 0.025$)$, and the levels
\end{abstract}


of miRNA-122 expression were significantly higher $(\mathrm{P}=0.008$ and $0.003)$ than those in the blank group. Compared with the blank group, apoptosis of Huh7 and HepG2 cells was significantly increased ( $\mathrm{P}=$ 0.001 and 0.027 ). We concluded that the expression of miRNA-122 is regulated by DNA methylation and correlated with apoptosis of liver cancer cells. Methylation regulation of miRNA-122 expression might be involved in the development of hepatocellular carcinoma.

Key words: MicroRNA-122; Methylation regulation; Apoptosis; Hepatocellular carcinoma

\section{INTRODUCTION}

Hepatocellular carcinoma (HCC) is one of the most common cancers in the world. It is frequently diagnosed at an advanced stage, resulting in poor survival rates. Although HCC has been widely studied for many years, its precise mechanism of development remains unknown. Similar to other tumors, HCC seems to occur via a multistep process, with an accumulation of genetic and epigenetic alterations in regulatory genes or mechanisms leading to the activation of oncogenes and inactivation of tumor suppressor genes (Nishida and Goel, 2011). An urgent need for better understanding of the mechanisms underlying HCC development and progression clearly exists, as it might help in the design of new strategies for detection and treatment.

Deregulation of the epigenome has been suggested to play an important role in tumor development and progression, especially DNA methylation and microRNA (miRNA) regulation (Lee et al., 2003). Aberrant methylation of many miRNA genes within or adjacent to the $\mathrm{CpG}$ island might result in the abnormal expression of miRNA and cause tumorigenesis (Weber et al., 2007). MiRNA-122 displays liver-specific expression essential to many liver physiological and pathological processes (Young et al., 2010; Jopling, 2012). Some studies have reported that miRNA-122 expression in HCC is low and that expression levels are positively correlated with the degree of differentiation and prognosis of HCC (Kutay et al., 2006; Lewis and Jopling, 2010).

Downregulation of cell or tissue miRNA-122 expression enhances the expression or development of tumors associated with target genes such as cyclin G1, the anti-apoptotic Bcl-w protein, and metalloproteinase 17 (Gramantieri et al., 2007; Lin et al., 2008). However, miRNA-122 expression varies by source of human liver tissue and liver cell lines (Narbus et al., 2011). Infection with the hepatitis B and C viruses is highly correlated with HCC. Research has shown that liver tissues infected with these viruses exhibit significantly lower and elevated levels of miRNA-122 expression, respectively (Varnholt et al., 2008).

Hepatocyte nuclear factors (HNFs) are important transcription factors in liver tissue. Several studies have demonstrated that HNF-4 and HNF- 6 are positive regulators of the miRNA-122 promoter (Li et al., 2011; Laudadio et al., 2012). In addition, DNA methylation is one of the main mechanisms of epigenetic regulation. Using methylation polymerase chain reaction (PCR), Jung et al. (2011) have reported that miRNA-122 expression might be associated with methylation of the miRNA-122 promoter. The methylation site, degree of methylation of the miRNA-122 promoter region, function, and value of methylation of the miRNA-122 promoter in HCC are unclear. Therefore, to explore the effects of epigenetic regulation on miRNA-122 expression, we investigated the methylation regulation of liver-specific miRNA-122 expression using methylation sequencing and 
miRNA quantitative assay. The effects of the regulation of DNA methylation of miRNA-122 on the proliferation and apoptosis of HCC cells were measured with flow cytometry and Cell Counting Kit-8 (CCK-8; Colleagues Chemistry Research Institute, Japan) assays. The mechanisms of action of miRNA-122 methylation in the development of HCC were analyzed.

\section{MATERIAL AND METHODS}

\section{Cell lines and primary tumor samples}

Two liver cancer cell lines (Huh7 and HepG2) and a normal liver cell line (QSG7701) were purchased from the Shanghai Institutes for Biological Sciences of the Chinese Academy of Sciences. All cell lines were cultured in accordance with manufacturer instructions. Human primary hepatocytes (hPHs) were obtained by separating normal liver tissue from resected specimens from patients with extrahepatic metastasis of HCC treated at Taizhou People's Hospital (Taizhou, China). The liver tissue specimens were confirmed by pathology.

\section{DNA demethylation of cells}

In this study, $10^{5} \mathrm{Huh} 7, \mathrm{HepG}$, and QSG-7701 cells were grown on 6-well plates and treated with various concentrations $(0,5$, and $10 \mu \mathrm{M})$ of 5-aza-2'-deoxycytidine (5-aza-dc; Sigma, USA) for 3 days. Three wells were used for each kind of processing. The methylation status of the miRNA-122 promoter was detected using a methylation sequencing method after $72 \mathrm{~h}$. The levels of miRNA-122 expression were measured using real-time quantitative PCR. Logarithmic growth phase cells were used in the experiments.

\section{Detection of miRNA-122 promoter methylation}

\section{DNA extraction and amplification}

The miRNA-122 promoter sequence is located in the chr18:54,264,000-54,265,000 region (Jung et al., 2011). One pair of bisulfite PCR primers was designed and synthesized, and the primer sequences are shown in Table 1. Primer synthesis was conducted by Sangon Biotech (Shanghai), Co., Ltd. (China). DNA was extracted from cells. Bisulfite modification of genomic DNA was performed using a methylation reagent kit (EZ DNA Methylation-Gold ${ }^{\mathrm{TM}}$ Kit, California Zymo Research, USA). Samples of DNA processed with bisulfate sequencing PCR primers were amplified using PCR. The length of the amplified PCR product was $486 \mathrm{bp}$. Figure 1 shows a part diagram of the gel electrophoresis process.

\section{Methylation sequencing and analysis of the PCR product}

PCR products of gel recycling were connected with a pMD19-T vector (Takara Co., Japan). Ten microliters of connected products was transformed into DH5a competent cells, coated with X-gal/isopropyl $\beta$-D-1-thiogalactopyranoside and an ampicillin agar (Luria-Bertani) plate, and incubated at $37^{\circ} \mathrm{C}$ overnight. Five white clones selected through blue/white screening were inoculated in $3 \mathrm{~mL}$ Luria-Bertani medium and incubated at $37^{\circ} \mathrm{C}$ overnight. 
Plasmids were extracted using an AxyPrep Plasmid Miniprep Kit (Axygen Company, USA). DNA sequencing was completed by Sangon Biotech. Analytical results of the degree of methylation of each sample are available at http://quma.cdb.riken.jp (Figure 2).

\section{Table 1. BSPCR primer sequence.}

\begin{tabular}{llc}
\hline Primer name & Primer sequence & Product (bp) \\
\hline $520-\mathrm{F}$ & 5'-TAGAAATTTGGGGAGGTGATTA-3' & 486 \\
$520-\mathrm{R}$ & 5'-CCAAAAAACRACCTAATCACTC-3 & \\
\hline
\end{tabular}

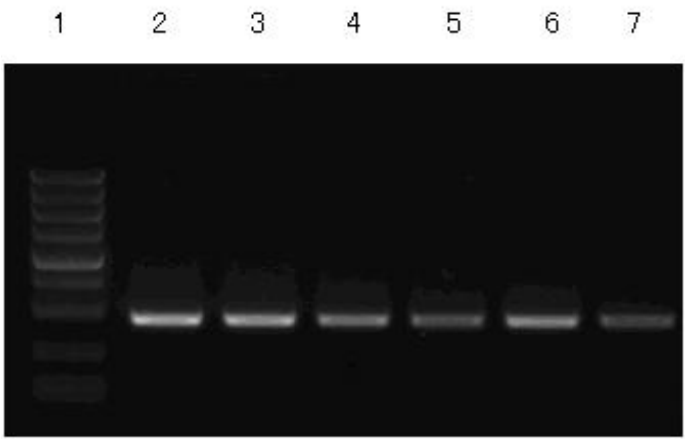

Figure 1. Products of PCR amplification. Lane 1 = Marker DL5000 (from upper to down: 5000, 3000, 2000, 1500, 1000, 750, 500, 250, 100 bp). Lanes 2 to 7 = Products of PCR amplification in Huh7, HepG2, Huh7-5D, HepG25D, QSG7701, QSG7701-5D (objective fragment $=486$ bp).

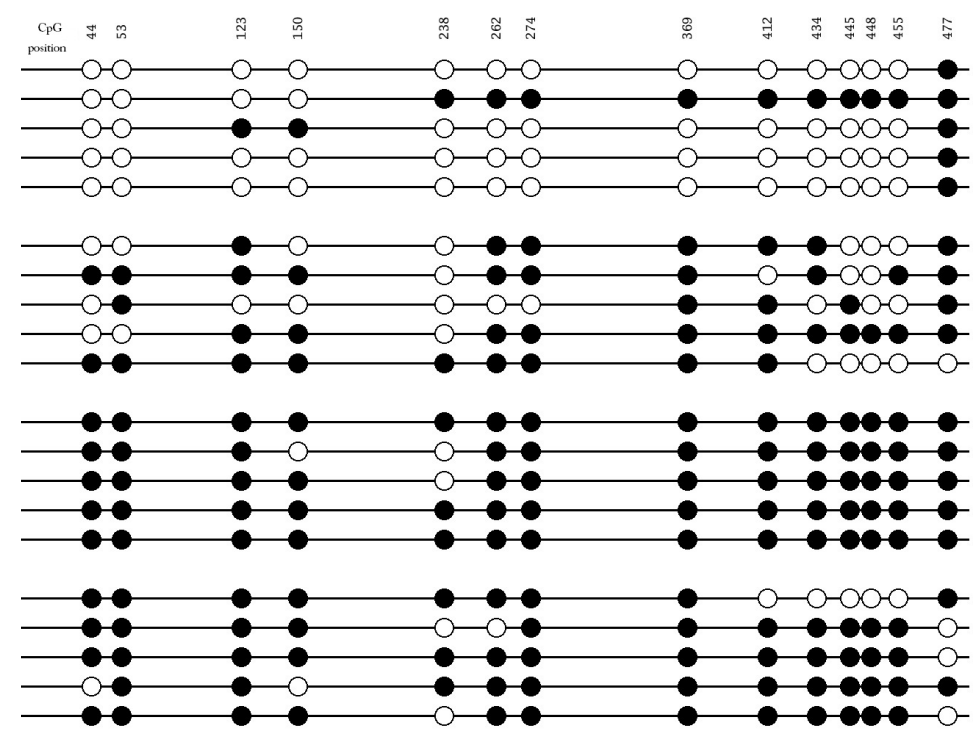

Figure 2. $\mathrm{CpG}$ sites of miRNA-122 promoter methylation. Each circle represents a CG; black circles represent methylation; white circles represent unmethylation. Each row represents the sample of a clone sequencing result. From top to bottom: lines 1 to $5=$ normal liver cells; lines 6 to $10=$ QSG-7701 cells; lines 11 to $15=$ HepG2 cells; lines 16 to $20=$ Huh7 cells. 


\section{Quantitative detection of miRNA-122}

Levels of miRNA-122 expression were detected via SYBR Green dye (Invitrogen Company, USA) assay. Total cellular RNA was extracted with TRIZOL (Invitrogen Company, USA) in a 1-step extraction procedure. Hsa-miRNA-122 and U6 reverse transcription primers and an amplification primer were designed and synthesized. The specific sequences are shown in Tables 2 and 3. Complementary DNA synthesis was performed with reverse transcription. PCR was completed with a real-time fluorescent quantitative PCR instrument (ABI 7300, Applied Biosystems, USA) under the following conditions: $95^{\circ} \mathrm{C}$ for $10 \mathrm{~min}$ and 40 cycles of $95^{\circ} \mathrm{C}$ for $10 \mathrm{~s}$ and $60^{\circ} \mathrm{C}$ for $60 \mathrm{~s}$. U6 was used as a reference. Data were analyzed using the $2-\Delta \Delta \mathrm{CT}$ method.

Table 2. Hsa-miR-122 and U6 RT primer.
\begin{tabular}{ll}
\hline Gene name & RT primer \\
\hline U6 & 5'-CGCTTCACGAATTTGCGTGTCAT-3' \\
hsa-miR-122 & 5'-GTCGTATCCAGTGCGTGTCGTGGAGTCGGCAATTGCACTGGATACGACcaaaca-3' \\
\hline
\end{tabular}

\begin{tabular}{|c|c|c|}
\hline Gene name & Primer sequence & Product (bp) \\
\hline \multirow[t]{2}{*}{$\overline{\mathrm{U} 6}$} & F: 5'-GCTTCGGCAGCACATATACTAAAAT-3' & \\
\hline & R: 5'-CGCTTCACGAATTTGCGTGTCAT-3' & 89 \\
\hline \multirow[t]{2}{*}{ hsa-miR-122 } & GSP: 5'-GGGTGGAGTGTGACAATGG-3' & \\
\hline & R: 5'-CAGTGCGTGTCGTGGAGT-3' & 65 \\
\hline
\end{tabular}

\section{Detection of cell proliferation activity}

Cell proliferation in the 3 cell lines was measured using CCK-8 (Colleagues Chemistry Research Institute) assay. The main steps were as follows: 2000 cells were grown in each well. CCK-8 solutions $(10 \mu \mathrm{L})$ were added to each well, and the reaction was incubated for $1 \mathrm{~h}$. Absorbance at $450 \mathrm{~nm}$ was measured using a microplate reader. A reaction that excluded cells but used corresponding amounts of cell culture solution and CCK-8 solution served as a blank control.

\section{Detection of cell apoptosis}

Cell apoptosis was detected using Annexin V fluorescein isothiocyanate (Merck Company, USA) and propidium iodide as follows: adjustment of cell density to approximately $1 \times 10^{6}$ cells $/ \mathrm{mL}$, medium removal, and washing with phosphate-buffered saline twice. The cells were gently suspended with $0.5 \mathrm{~mL}$ precooled $1 \mathrm{X}$ binding buffer and added to $1.25 \mu \mathrm{L}$ Annexin V fluorescein isothiocyanate. The photophobic reactions were incubated at room temperature for $15 \mathrm{~min}$. Supernatant was removed after $1000 \mathrm{x} \mathrm{g}$ centrifugation for $5 \mathrm{~min}$. The cells were gently suspended with $0.5 \mathrm{~mL}$ precooled $1 \mathrm{X}$ binding buffer and mixed with $10 \mu \mathrm{L}$ propidium iodide. The samples were placed on ice, stored away from light, and immediately detected with a flow cytometer (BD Company, USA; Figure 3). 

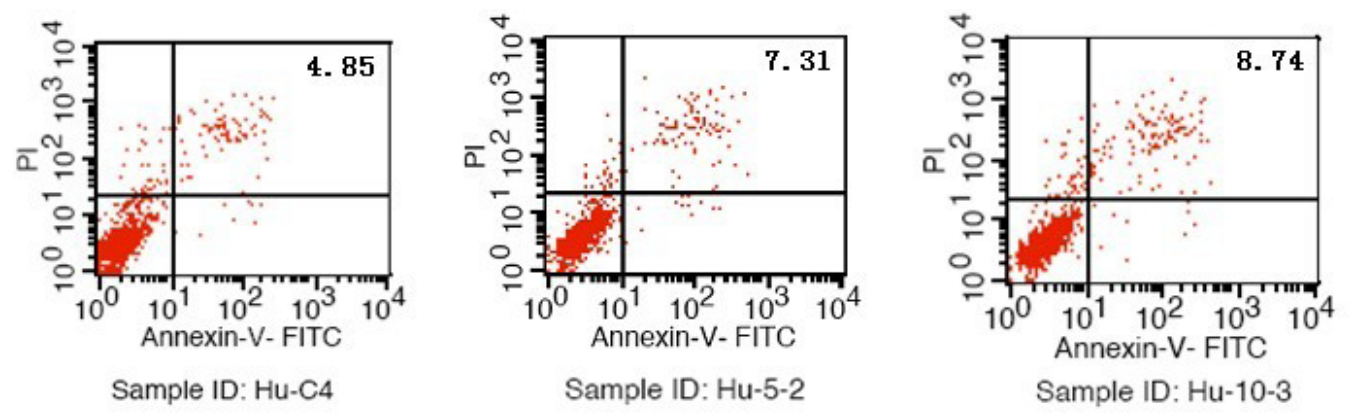

Figure 3. Cell apoptosis by flow cytometry.

\section{Statistical analysis}

All values were expressed as means \pm standard deviation. Data analysis was conducted using SPSS 17.0 (SPSS Inc., Chicago, IL, USA). One-way analysis of variance and the Student-Newman-Keuls $q$-test were used among the groups. Student's $t$-test was used for independent data. A P value of $<0.05$ was considered statistically significant.

\section{RESULTS}

\section{Methylation of the miRNA-122 promoter and expression of miRNA-122 in various liver cells}

The methylation status of the miRNA-122 promoter of the 4 cell types investigated in this study was measured via DNA methylation sequencing. The level of miRNA-122 expression was detected with real-time quantitative PCR. Compared with that of the hPHs $(21.9 \pm 11.4 \%)$, the degree of methylation of the miRNA-122 promoter of the Huh7 (87.6 $\pm 9.3 \%)$, HepG2 (89 $\pm 14.3 \%)$, and QSG-7701 $(69.5 \pm 11.5 \%)$ cell lines was significantly increased $(F=25.9, \mathrm{P}=$ 0.000 ), especially in the Huh7 and HepG2 cell lines. The levels of miRNA-122 expression in the Huh7, HepG2, and QSG-7701 cell lines were significantly lower than that in hPHs $(2.83 \mathrm{x}$ $\left.10^{4} \pm 3746\right)$, especially in the Huh7 and HepG2 cell lines $(F=12.9, \mathrm{P}=0.007$; Table 4$)$.

\begin{tabular}{|c|c|c|c|c|}
\hline & Huh7 cell & HepG2 cell & QSG-7701 cell & $\mathrm{hPH}$ \\
\hline $\begin{array}{l}\text { Methylation (\%) } \\
\text { miR-122 }\end{array}$ & $\begin{array}{l}87.6 \pm 9.3^{\#} \\
0.99 \pm 0.08^{*}\end{array}$ & $\begin{array}{l}91.1 \pm 10.9^{*} \\
1.14 \pm 0.11^{*}\end{array}$ & $\begin{array}{l}69.5 \pm 11.6^{\#} \\
1.60 \pm 0.23\end{array}$ & $\begin{array}{r}21.9 \pm 11.4 \\
2.83 \times 10^{4} \pm 3746\end{array}$ \\
\hline
\end{tabular}

\section{Effects of demethylation treatment on miRNA-122 promoter methylation}

The Huh7, HepG2, and QSG-7701 cell lines were treated with various concentrations $(0,5$, and $10 \mu \mathrm{M})$ of 5-aza-dc. Compared with those of the blank group, the methylation levels of the miRNA-122 promoter in the Huh7 and HepG2 cell lines at $10 \mu \mathrm{M}$ were reduced, and 
the difference was significant $(F=5.91, \mathrm{P}=0.038 ; F=7.32, \mathrm{P}=0.025$, respectively). The methylation level of the miRNA-122 promoter in the QSG-7701 cell line slightly decreased, but the difference had no statistical significance $(F=1.03, \mathrm{P}=0.413$; Figure 4$)$.

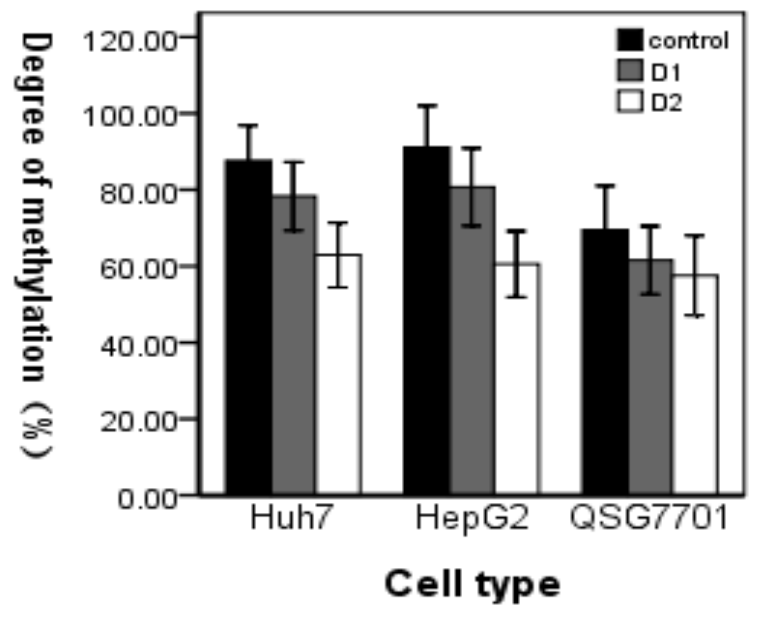

Figure 4. Effect of demethylation on miR-122 promoter methylation. Control = blank control; D1 = 5 $\mu \mathrm{M} 5 \mathrm{Aza}-\mathrm{dc}$; $\mathrm{D} 10=10 \mu \mathrm{M}$ of $5 \mathrm{Aza}-\mathrm{dc}$.

\section{Effects of demethylation treatment on hepatic cell miRNA-122 expression}

The Huh7, HepG2, and QSG-7701 cell lines were treated with various concentrations ( 0 , 5, and $10 \mu \mathrm{M})$ of 5-aza-dc. The levels of miRNA-122 expression in the Huh7 and HepG2 cell lines at $10 \mu \mathrm{M}$ were significantly elevated compared with that of the blank group $(F=11.9, \mathrm{P}=0.008$; $F=18.6, \mathrm{P}=0.003$, respectively). The level of miRNA-122 expression in the QSG-7701 cell line decreased, but the difference had no statistical significance $(F=1.54, \mathrm{P}=0.289$; Figure 5).

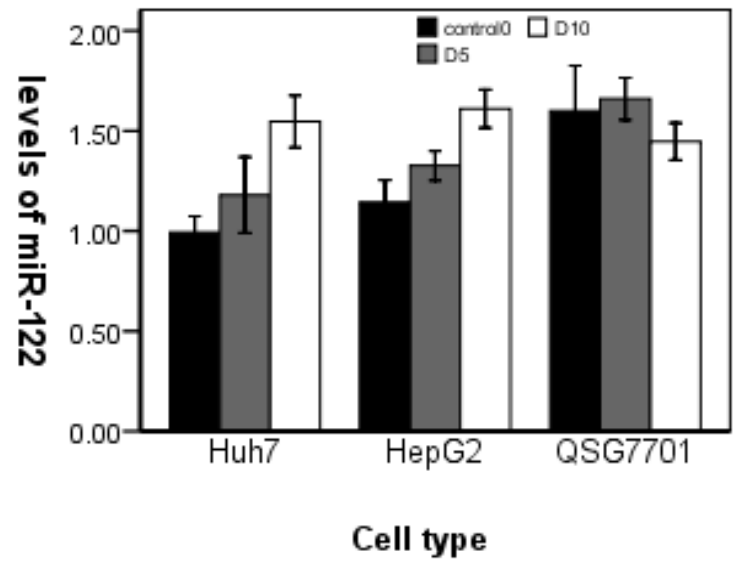

Figure 5. Effect of demethylation on miR-122 expression. Control $0=$ blank control; D5 = $5 \mu \mathrm{M}$ 5Aza-dc; D10 = $10 \mu \mathrm{M} 5$ Aza-dc. 


\section{Hepatic cell proliferation after treatment with a demethylation agent}

The Huh7, HepG2, and QSG-7701 cell lines were treated with various concentrations $(0,5$, and $10 \mu \mathrm{M})$ of 5-aza-dc. Cell proliferation was detected with CCK-8. Compared with that in the blank control, the proliferation of Huh7 and HepG2 cells after treatment was slightly elevated, and that of QSG-7701 cells was slightly decreased, but the difference had no statistical significance $(\mathrm{P}=0.125,0.154$, and 0.237 , respectively; Table 5).

\begin{tabular}{|c|c|c|c|}
\hline Concentration $(\mu \mathrm{M})$ & Huh7 cell" & HepG2 cell* & QSG-7701 cell \\
\hline 0 & $1.18 \pm 0.21$ & $1.59 \pm 0.23$ & $1.36 \pm 0.11$ \\
\hline 5 & $1.45 \pm 0.10$ & $1.85 \pm 0.06$ & $1.44 \pm 0.02$ \\
\hline 10 & $1.37 \pm 0.03$ & $1.74 \pm 0.05$ & $1.21 \pm 0.23$ \\
\hline
\end{tabular}

\section{Effects of demethylation treatment on hepatic cell apoptosis}

The Huh7, HepG2, and QSG-7701 cell lines were treated with various concentrations $(0,5$, and $10 \mu \mathrm{M})$ of 5-aza-dc. Cell apoptosis in advanced cells was detected using flow cytometry. Compared with that in the blank control, the level of apoptosis in Huh7 and HepG2 cells after treatment increased significantly $(\mathrm{P}=0.001, \mathrm{P}=0.027$, respectively). No significant differences were found between treatment with 5 and $10 \mu \mathrm{M}$ of 5-aza-dc in Huh7 and HepG2 cells. The levels of apoptosis in QSG-7701 cells before and after treatment were not significantly different $(\mathrm{P}=0.347$; Table 6$)$.

Table 6. Effect of demethylation treatment on hepatic cells apoptosis.
\begin{tabular}{lccc}
\hline Concentration $(\mu \mathrm{mol} / \mathrm{L})$ & Huh7 cell ${ }^{\#}$ & HepG2 cell & QSG-7701 cell $^{\S}$ \\
\hline 0 & $5.43 \pm 0.54$ & $6.42 \pm 0.71$ & $5.68 \pm 1.47$ \\
5 & $7.67 \pm 1.35$ & $7.15 \pm 0.09$ & $4.60 \pm 1.65$ \\
10 & $8.72 \pm 0.88$ & $8.36 \pm 1.07$ & $4.35 \pm 0.48$ \\
\hline${ }^{\#} F=8.85, \mathrm{P}=0.016 ; * F=5.50, \mathrm{P}=0.027 ;{ }^{\S} F=1.18, \mathrm{P}=0.347$. &
\end{tabular}

\section{DISCUSSION}

The expression of miRNA has spatiotemporal characteristics and it is regulated by transcription factors and DNA methylation, among other factors (Pitto et al., 2008). As a small RNA specific to liver tissue, miRNA-122 is more highly expressed in normal liver cells than in HCC tissues (Kutay et al., 2006; Xu et al., 2012). The miRNA-122 precursor gene promoter is located on its stem-loop sequence approximately $5 \mathrm{~kb}$ upstream and contains multiple nuclear receptor binding sequences and promoter cis-acting elements (Li et al., 2011). The role of DNA methylation in miRNA-122 expression must be further elucidated. In this study, the methylation level of the miRNA-122 promoter region in hPHs was lower than those in the Huh7 and HepG2 cell lines. The level of miRNA-122 expression in hPHs was significantly higher than those in the Huh7 and HepG2 cell lines. These results are consistent with data reported by Jung et al. (2011). The methylation level of the QSG-7701 cells increased, whereas 
their miRNA-122 levels significantly decreased. The degree of methylation and miRNA-122 levels of the QSG-7701 cells were lower than those of the Huh7 and HepG2 cells, suggesting that miRNA-122 expression is regulated by promoter methylation. A decreasing pattern of miRNA-122 expression in normal hepatocytes and normal liver and hepatoma cell lines was observed, indicating that miRNA-122 might be involved in HCC development.

5-Aza-dc is a commonly used demethylating agent. The degree of methylation in the QSG-7701, HepG2, and Hep3B cells significantly decreased, whereas their levels of miRNA-122 expression markedly increased after treatment with 5-aza-dc. These results further confirmed that the expression of miRNA-122 was regulated by DNA methylation. However, the effect of 5-aza-dc on the methylation of the QSG-7701 cell line was subtle and might be associated with its low methylation. Fourteen methylation sites were identified in this study. Only 477 sites (86.7\%) exhibited high methylation in hPHs, whereas only 123, 262, 274, 369, and 412 sites demonstrated high methylation in the QSG-7701 cell line. In contrast, 238 sites displayed low methylation in the HepG2 cell line; the rest exhibited high methylation. Only 238 and 477 sites demonstrated low methylation in the Huh7 cell line, with the rest displaying high methylation. These results suggest that the methylation sites of the miRNA-122 promoter in different cells vary and warrant further investigation for biological significance.

Several reports have shown that the downregulation of miRNA-122 expression leads to the development of tumors associated with target genes such as cyclin G1 and the antiapoptotic protein Bcl-w. A reduction in miRNA-122 expression can cause rapid cancerous cell proliferation (Kutay et al., 2006; Gramantieri et al., 2007), and enhanced expression of miRNA-122 might inhibit intracellular Bcl-w expression and decrease cell viability (Lin et al., 2008). No significant alterations were found in the proliferation of Huh7, HepG2, and QSG7701 cells treated with 5-aza-dc in the present study; however, miRNA-122 expression levels were significantly elevated, which is less consistent with previous reports (Gramantieri et al., 2007; Wu et al., 2009). The reasons for this difference may be associated with the low levels of miRNA-122 expression in the 3 hepatic cell lines themselves; the levels of miRNA-122 elevation were limited after demethylation treatment and insufficient to induce cell proliferation-related changes. In addition, the difference in detection methods and application of cell lines cannot be excluded. The apoptosis of Huh7 and HepG2 cells significantly increased via the demethylation process in the present study, which suggested that the demethylation of the miRNA-122 promoter has obvious effects on apoptosis in hepatoma cells.

In conclusion, liver-specific miRNA-122 expression is regulated by DNA methylation, which is closely related to hepatoma cell apoptosis. This relationship suggests that miRNA-122 methylation regulation might be involved in the development of liver cancer.

\section{Conflicts of interest}

The authors declare no conflict of interest.

\section{REFERENCES}

Gramantieri L, Ferracin M, Fornari F, Veronese A, et al. (2007). Cyclin G1 is a target of miR-122a, a microRNA frequently down-regulated in human hepatocellular carcinoma. Cancer Res. 67: 6092-6099.

Jopling C (2012). Liver-specific microRNA-122: Biogenesis and function. RNA. Biol. 9: 137-142.

Jung CJ, Iyengar S, Blahnik KR, Ajuha TP, et al. (2011). Epigenetic modulation of miR-122 facilitates human embryonic 
stem cell self-renewal and hepatocellular carcinoma proliferation. Plos One 6: e27740.

Kutay H, Bai S, Datta J, Motiwala T, et al. (2006). Downregulation of miR-122 in the rodent and human hepatocellular carcinomas. J. Cell Biochem. 99: 671-678.

Laudadio I, Manfroid I, Achouri Y, Schmidt D, et al. (2012). A feedback loop between the liver-enriched transcription factor network and miR-122 controls hepatocyte differentiation. Gastroenterology 142: 119-129.

Lee S, Lee HJ, Kim JH, Lee HS, et al. (2003). Aberrant CpG island hypermethylation along multistep hepatocarcinogenesis. Am. J. Pathol. 163: 1371-1378.

Lewis AP and Jopling CL (2010). Regulation and biological function of the liver-specific miR-122. Biochem. Soc. Trans. 38: 1553-1557.

Li ZY, Xi Y, Zhu WN, Zeng C, et al. (2011). Positive regulation of hepatic miR-122 expression by HNF4alpha. J. Hepatol. 55: 602-611.

Lin CJ, Gong HY, Tseng HC, Wang WL, et al. (2008). miR-122 targets an anti-apoptotic gene, Bcl-w, in human hepatocellular carcinoma cell lines. Biochem. Biophys. Res. Commun. 375: 315-320.

Narbus CM, Israelow B, Sourisseau M, Michta ML, et al. (2011). HepG2 cells expressing microRNA miR-122 support the entire hepatitis C virus life cycle. J. Virol. 85: 12087-12092.

Nishida N and Goel A (2011). Genetic and epigenetic signatures in human hepatocellular carcinoma: a systematic review. Curr. Genomics 12: 130-137.

Pitto L, Ripoli A, Cremisi F, Simili M, et al. (2008). microRNA(interference) networks are embedded in the gene regulatory networks. Cell Cycle 7: 2458-2461.

Varnholt H, Drebber U, Schulze F, Wedemeyer I, et al. (2008). MicroRNA gene expression profile of hepatitis C virusassociated hepatocellular carcinoma. Hepatology 47: 1223-1232.

Weber B, Stresemann C, Brueckner B and Lyko F (2007). Methylation of human microRNA genes in normal and neoplastic cells. Cell Cycle 6: 1001-1005.

Wu X, Wu S, Tong L, Luan T, et al. (2009). miR-122 affects the viability and apoptosis of hepatocellular carcinoma cells. Scand. J. Gastroenterol. 44: 1332-1339.

Xu J, Zhu X, Wu L, Yang R, et al. (2012). MicroRNA-122 suppresses cell proliferation and induces cell apoptosis in hepatocellular carcinoma by directly targeting Wnt/beta-catenin pathway. Liver Int. 32: 752-760.

Young DD, Connelly CM, Grohmann C and Deiters A (2010). Small molecule modifiers of microRNA miR-122 function for the treatment of hepatitis C virus infection and hepatocellular carcinoma. J. Am. Chem. Soc. 132: 7976-7981. 